\title{
Histochemical staining and quantification of arbuscular mycorrhizal fungal colonization
}

Tania Ho-Plágaro, María Isabel Tamayo-Navarrete and José Manuel García-Garrido

Department of Soil Microbiology and Symbiotic Systems, Estación Experimental del Zaidín

(EEZ) CSIC, Calle Profesor Albareda no1, 18008 Granada, Spain

*Corresponding author: Dr. J. M. García-Garrido: josemanuel.garcia@eez.csic.es

Running Head: AM histochemical staining and quantification 


\title{
Histochemical staining and quantification of arbuscular mycorrhizal fungal colonization
}

Tania Ho-Plágaro, María Isabel Tamayo-Navarrete and José Manuel García-Garrido

\begin{abstract}
Histochemical staining and light microscopy-based techniques have been widely used to detect and quantify Arbuscular Mycorrhizal fungi (AMF) in roots. Here we describe a standardized method for staining of AMF in colonized roots, and we provide possible modifications to adjust the protocol according to particular requirements, such as the type of root material or the reduction of toxic products. In addition, we also summarize some of the most common ways to quantify arbuscular mycorrhizal colonization.
\end{abstract}

Key words Arbuscular mycorrhizal roots, Non-vital staining, Trypan blue, AM quantification

\section{Introduction}

Regardless of the main objective of each investigation in arbuscular mycorrhiza (AM) research, the techniques to detect and quantify arbuscular mycorrhizal fungi (AMF) in roots are still essential tools in mycorrhizal research. Historically, light microscopy-based techniques have been used to detect and quantify AMF in roots, including both, non-vital staining methods and vital root staining methods (reviewed by Vierheiling et al., 2005 [1]). More recently, fluorescent staining of arbuscular mycorrhizal structures coupled with biochemical methods 
that measure characteristic biochemical markers for AMF or the AM symbiosis [2-4] and recent developed quantitative and qualitative molecular tools $[5,6]$ have become reliable tools for the identification and/or quantification of $\mathrm{AMF}$ in roots and thus a serious alternative or complement to root staining.

In this chapter, we describe a generalized light microscopy-based procedure to quantify AMF in roots after convectional histochemical staining, excluding vital staining or fluorescent staining. This method is still used as the standard technique for the measurement of the degree of root colonization by AMF and to identify and count the fungal structures in the root. We provide some modifications and adaptations in both, staining and quantification methods in order to help the reader to choose the most appropriate procedure to quantify AMF in roots for particular purposes.

\section{Materials}

\subsection{Plant Material}

Plant roots colonized by arbuscular mycorrhizal fungi (AMF). Although the present protocol is adjusted for tomato roots, AMF staining can be performed in many different plant species. Recommended modifications will be shown in order to adapt the protocol to thinner or thicker roots (see Notes 1-3).

\subsection{Solutions}

1. Clearing solution: $10 \% \mathrm{KOH}$. Add about $100 \mathrm{~mL}$ of water to a glass beaker. Weigh $100 \mathrm{~g}$ of $\mathrm{KOH}$. Add water to a volume of $1000 \mathrm{~mL}$. (Note: Bases should not be stored in bottles with ground glass stoppers). 
2. Acidifying solution: $0.1 \mathrm{~N} \mathrm{HCl}$ solution. Concentrated $\mathrm{HCl}$ is approximately $37 \%$. Add $9.85 \mathrm{~mL}$ of concentrated $\mathrm{HCl}$ in $990 \mathrm{~mL}$ of water.

3. Staining solution: $0.05 \%$ Trypan blue in lactic acid. Dissolve $0.5 \mathrm{~g}$ of Trypan blue in $1 \mathrm{~L}$ of lactic acid. Store in a glass bottle.

4. Destaining solution and mounting media: lactic acid.

\subsection{Containers}

1. Biopsy plastic cassettes. Pore size of the cassettes depends on the thickness of the stained roots, but the best for root samples are those with square holes in dimensions of $0.9 \mathrm{~mm} \times 0.9 \mathrm{~mm}$. Alternatively, Falcon tubes can also be used to immerse the roots directly into the solutions, but in this case washes and solution changes are more tedious.

2. Glass beakers

3. Plastic or glass vials with tight-sealing lids for storage of samples

4. Clear plastic dishes with inscribed grid lines to measure colonization

\subsection{Equipment}

1. Fine forceps for manipulating roots

2. Fume hood

3. Steel cooking pot

4. Portable electric stove

5. Microscope slides, long cover slips

6. Dissecting microscope with a transmitted light illumination - a clear plastic panel over the microscope base is recommended to provide a stable platform and for protection from spilled liquids

7. Counter 


\section{Methods}

\subsection{Histochemical staining}

The non-vital trypan blue histochemical staining is performed following the method described by Phillips and Hayman [7] with some modifications.

1. Carefully wash soil from roots and cut them into $1 \mathrm{~cm}$ long segments (see Note 4).

2. In a plastic cassette, set up the root segments. Do not fill the cassettes with too many roots to allow maximum exposure of the roots to the solutions and a proper staining. Place the cassettes in a beaker with tap water until staining. If convenient, roots can be kept at $4^{\circ} \mathrm{C}$ for staining the next day.

3. Prepare a boiling water bath by heating water in a steel cooking pot using a portable electric stove placed inside a fume hood (see Note 5).

4. Remove the tap water from the roots, cover them with $10 \% \mathrm{KOH}$ and set the beaker in the boiling water bath during 10 min (approximately, depending on the hardness of the root (see Notes 1-3). This step removes the cell root cytoplasm and most of the nuclei, and the roots become very clear with the vascular cylinder distinctly visible.

5. Discard the $\mathrm{KOH}$ and rinse a few times with tap water

6. Once drained, cover the roots with $0.1 \mathrm{~N} \mathrm{HCl}$ during 3 min at room temperature. The acid neutralizes the base $\mathrm{KOH}$, so the stain can react with the tissues.

7. Decant the $\mathrm{HCl}$ solution (without rinsing) and cover the roots with $0.05 \%$ Trypan Blue in lactic acid during 10-15 minutes at boiling temperature (see Notes 6-7) 
8. Pour off as much of the colorant as possible and cover the cassettes with lactic acid. This solution will remove the excessive stain and will preserve the roots until its microscopic observation (see Notes 7-8).

9. Roots with lactic acid can be spread out in a Petri dish for its visualization with a stereomicroscope (see Note 9). For a microscopic observation, mount root pieces on a microscope slide with clear lactic acid (see Note 10). Gently push the cover slip onto the root to flatten.

3.2 Examination and quantification by the gridline intersect method

As a first screening, mycorrhiza development is usually evaluated in terms of percentage of mycorrhizal root length by the gridline intersect method described by Giovannetti and Mosse [8]. This method is used to estimate the proportion of total colonized and uncolonized root length. The counting of the colonized and uncolonized roots is summarized in Fig. 1.

1. A Petri dish is marked with a grid line following two requirements:

- $\quad$ The grid of lines must form squares of $1.27 \mathrm{~cm} \times 1.27 \mathrm{~cm}$ dimension. In this manner, based on the formula of Newman [9], the total number of intersects between grid lines and roots will equal the total root length in $\mathrm{cm}$, so we can express the total colonized root in terms of length.

- The position of the grid squares relative to the container should be set so that the edge of the container must not coincide with a grid line, but the two partial squares at each edge together make a complete square.

2. The root sample is spread out evenly in the grid-lined Petri dish. 
3. Under a stereomicroscope, vertical and horizontal gridlines are scanned and the presence or absence of colonization is recorded at each point where the roots intersect a line. If possible, at least 200 root-gridline intersects are recorded. The use of a counter might facilitate this task.

4. Percentage of mycorrhizal colonization is calculated as indicated in the formula of Fig. 1.

\subsection{Examination and quantification by morphometric procedures}

In order to measure in a much more accurate way the fungal structures and the lifespan of active mycorrhizal associations in the roots, other techniques have been developed, named as morphometric procedures. These procedures allow the determination of the area or length of structures using a microscope. Here, we will explain two of the most widely used morphometric methods, the first one developed by Trouvelot et al in 1986 [10] and the second one from McGonigle et al. in 1990 [11].

\subsubsection{The Trouvelot morphometric method}

1. Per biological replicate, three microscope slides are prepared, each slide containing thirty $1-\mathrm{cm}$ root pieces.

2. Using a microscope, mycorrhizal intensity and arbuscule abundance of every root fragment is rated according to the range of classes indicated in Fig. 2.

3. For each biological replicate, the obtained rate values are used to calculate the mycorrhization parameters through the following formulas:

\section{- Frequency of mycorrhiza in the root system}

Frequency of colonization $(\mathrm{F} \%):=$ (Number of mycorrhized fragments/Number of total fragments) $* 100$ 
- Intensity of the mycorrhizal colonization in the entire root system

Relative mycorrhizal root length, relative mycorrhizal intensity $(\mathrm{M} \%)=$ $\left(95 * n_{5}+70 * n_{4}+30 * n_{3}+5 * n_{2}+n_{1}\right) /($ number of total fragments) where $n_{5}=$ number of fragments rated 5 according to mycorrhizal colonization; $n_{4}=$ number of fragments rated 4 etc.

- Intensity of the mycorrhizal colonization in the root fragments Absolute mycorrhizal root length, absolute mycorrhizal intensity (m\%) $=\mathrm{M} \%{ }^{*}$ (number of total fragments)/(number of mycorrhized fragments)

\section{- Arbuscule abundance in mycorrhizal parts of root fragments}

Absolute arbuscule richness $(a \%)=(100 * \mathrm{~mA} 3+50 * \mathrm{~mA} 2+10 * \mathrm{~mA} 1) / 100$

where $\mathrm{mA} 3, \mathrm{~mA} 2, \mathrm{~mA} 1$ are the $\%$ of $m$, rated $\mathrm{A} 3, \mathrm{~A} 2, \mathrm{~A} 1$, respectively, with

$m A 3=\left(\left(95 * n_{5} A 3+70 * n_{4} A 3+30 * n_{3} A 3+5 * n_{2} A 3+n_{1} A 3\right) /\right.$ number of mycorrhized fragments)*100/m and the same for $\mathrm{A} 2$ and $\mathrm{A} 1$.

Where e. g. $\mathrm{n}_{5} \mathrm{~A} 3=$ number of fragments rated 5 according to mycorrhizal colonization and rated 3 depending on the arbuscule abundance

\section{- Arbuscule abundance in the entire root system}

Relative arbuscule richness (" $\mathrm{A} \%$ ") $=\mathrm{a} * \mathrm{M} / 100$

Alternatively, mycorrhizal parameters can be quantified using MYCOCALC software (http://www.dijon.inra.fr/mychintec/Mycocalc-prg/download.html).

\subsubsection{The McGonigle magnified intersections method (modified)}

While the Trouvelot et al. method [10] explained above is observer-dependent, McGonigle et al. [11] described an improved method to estimate mycorrhizal colonization in a more objective scale of measurement, which also eliminates the subjective interpretation of 
whether hyphae seen in the absence of arbuscules are mycorrhizal. A comparison between the two methods is illustrated in Fig. 3. The main steps of the McGonigle procedure, with slight modifications, are as follow:

1. Per biological replicate, three microscope slides are prepared, each slide containing thirty $1-\mathrm{cm}$ root pieces.

2. Through visualization of the samples at magnification 200x and using a microscope eyepiece crosshair reticule, the intersections with the root fragments are rated according to the presence or absence of arbuscules, vesicles and hyphae. If a microscope eyepiece crosshair reticule is not available, it is also possible to perform the counting by following imaginary lines perpendicular to roots. Note that the presence of arbuscules and/or vesicles ALWAYS implies the presence of hyphae (see Example in Fig. 3)

3. For each biological replicate, the obtained rate values are used to calculate the mycorrhization parameters through the following formulas:

- Proportion of root length containing arbuscules

Arbuscular colonization $(\mathrm{AC} \%)=$ (Number of intersections with arbuscules/Number of total intersections) $* 100$

- Proportion of root length containing vesicles

Vesicular colonization (VC\%) $=$ (Number of intersections with vesicles/Number of total intersections)*100

- Proportion of root length containing hyphae

Hyphal colonization $(\mathrm{HC} \%)=$ (Number of intersections with hyphae/Number of total intersections) $* 100$ 


\section{Notes}

1. The described protocol is adjusted for staining of tomato mycorrhizal roots or similar. However, trypan blue staining of AMF is also useful for the staining of AMF colonizing the roots from many other different plant species, from herbs to trees. The thicker is the root, the more we will need to increase the time of $\mathrm{KOH}$ and/or staining treatments.

2. If delicate tissues are destroyed by high temperature treatments, try soaking the tissue overnight in the $\mathrm{KOH}$ solution and/or the staining solution at room temperature. Also try this if you wish to decrease the blue background staining of the root.

3. If standardized times are insufficient for AM fungal staining of thicker roots, try to increase the exposure of the roots to the $\mathrm{KOH}$ and/or the staining treatments. For example, for maize 1 hour at $90 \circ \mathrm{C}$ in $10 \% \mathrm{KOH}$ may work [12], and for shrubs, such us Artemisa, we may need to increase both the $\mathrm{KOH}$ and the trypan blue treatments to 2-3 hours [13].

4. The roots used for the staining must be fresh, or kept for 1 day maximum at 4 으 immersed in water. Alternatively frozen material kept at $-80^{\circ} \mathrm{C}$ can be also used.

5. The use of a fume hood is highly recommended, as the boiling of $10 \% \mathrm{KOH}$ and lactic acid produces toxic vapours.

6. Potential health hazards are associated with Trypan Blue and it has been confirmed as an animal carcinogen by the International Agency for Research of Cancer (IARC) [14]. The most common alternatives to Trypan blue are Acid Fuchsin, Cotton Blue and Black Ink. This last option described by Vierheiling in 
1998 [15] also avoids the inhalation of irritating vapours derived from heating the lactic acid.

7. Inhalation of mist from lactic acid causes coughing and irritation of mucous membranes. In order to reduce the use of lactic acid, we can use lactoglycerol (1:1:1 lactic acid, glycerol and water) for the trypan blue solution and as an alternative destaining solution. It should be prepared with $33 \mathrm{~mL}$ of lactic acid, $33 \mathrm{~mL}$ of glycerol and $33 \mathrm{~mL}$ of water.

8. If the blue background is too high, try recommendations from Note 2, or change the destaining solution at least once.

9. For visualization of the roots it is better to wait at least one day, in order to allow the roots to release air bubbles that may hamper proper observation of AM fungal colonization.

10. For long storage of samples it is recommended to prepare permanent slides with Polyvinyl alcohol-lactic acid-glycerol (PVLG) [16]. This mounting media allows to obtain permanent slides that remain unchanged even after 20 years of storage. It is prepared by dissolving $16.6 \mathrm{~g}$ of polyvinyl alcohol in $100 \mathrm{~mL}$ of distilled water in a hot water bath at $70-80 \circ \mathrm{C}$, and then mixing the solution with $100 \mathrm{~mL}$ of lactic acid and $100 \mathrm{~mL}$ of glycerol.

\section{Acknowledgement}

This work was supported by grants from the Comisión Interministerial de Ciencia y Tecnología (CICYT) and Fondos Europeos de Desarrollo Regional (FEDER) through the Ministerio de Economía y Competitividad in Spain (AGL2017-83871-P). 


\section{References}

1. Vierheilig $H$, Schweiger $P$, Brundrett $M$ (2005) An overview of methods for the detection and observation of arbuscular mycorrhizal fungi in roots. Physiol Plant 125 (4):393-404

2. Bothe $H$, Klingner A, Kaldorf M, Schmitz O, Esch H, Hundeshagen B, Kernebeck $H$ (1994) Biochemical approaches to the study of plant-fungal interactions in arbuscular mycorrhiza. Experientia 50 (10):919-925

3. Frey B, Buser H, Schüepp H (1992) Identification of ergosterol in vesicular-arbuscular mycorrhizae. Biol Fertility Soils 13 (4):229-234

4. Smith S, Dickson S (1991) Quantification of active vesicular-arbuscular mycorrhizal infection using image analysis and other techniques. Funct Plant Biol 18 (6):637-648

5. Alkan N, Gadkar V, Coburn J, Yarden O, Kapulnik Y (2004) Quantification of the arbuscular mycorrhizal fungus Glomus intraradices in host tissue using real-time polymerase chain reaction. New Phytol 161 (3):877-885

6. Sanders IR (2002) Specificity in the arbuscular mycorrhizal symbiosis. In: Mycorrhizal ecology. Springer, pp 415-437

7. Phillips JM, Hayman D (1970) Improved procedures for clearing roots and staining parasitic and vesicular-arbuscular mycorrhizal fungi for rapid assessment of infection. Trans Br Mycol Soc 55 (1):158IN116-161IN118

8. Giovannetti M, Mosse B (1980) An evaluation of techniques for measuring vesicular arbuscular mycorrhizal infection in roots. New Phytol 84 (3):489-500

9. Newman E (1966) A method of estimating the total length of root in a sample. J Appl Ecol:139-145 
10. Trouvelot A (1986) Mesure du taux de mycorhization VA d'un systeme radiculaire.

Recherche de methodes d'estimation ayant une significantion fonctionnelle. Mycorrhizae: physiology and genetics:217-221

11. McGonigle T, Miller M, Evans D, Fairchild G, Swan J (1990) A new method which gives an objective measure of colonization of roots by vesicular-arbuscular mycorrhizal fungi. New Phytol 115 (3):495-501

12. Liu F, Xu Y, Han G, Wang W, Li X, Cheng B (2018) Identification and functional characterization of a maize phosphate transporter induced by mycorrhiza formation. Plant Cell Physiol 59 (8):1683-1694

13. Hu D, Baskin JM, Baskin CC, Wang Z, Zhang S, Yang X, Huang Z (2019) Arbuscular mycorrhizal symbiosis and achene mucilage have independent functions in seedling growth of a desert shrub. J Plant Physiol 232:1-11

14. Grace C, Stribley DP (1991) A safer procedure for routine staining of vesicular-arbuscular mycorrhizal fungi. Mycol Res 95 (10):1160-1162

15. Vierheilig H, Coughlan AP, Wyss U, Piché Y (1998) Ink and vinegar, a simple staining technique for arbuscular-mycorrhizal fungi. Appl Environ Microbiol 64 (12):5004-5007

16. KOSKE R, TESSIER B 1983. A convenient. permanent slide mounting medium. Mycol Soc Amer News Lett 34:59 


\section{Figure Captions}

Figure 1. The gridline intersection method of Giovannetti and Mosse [8].

Figure 2. Scoring scale to evaluate the level of mycorrhizal colonization and the arbuscule abundance in roots fragments. Figure modified from Trouvelot et al. [10].

Figure 3. Summary and comparison of the two morphometric procedures described in this chapter. 
Figure 1

Examine about 200 intersections between vertical and horizontal gridlines and roots, and count the number of intersections where the root is mycorrhized:

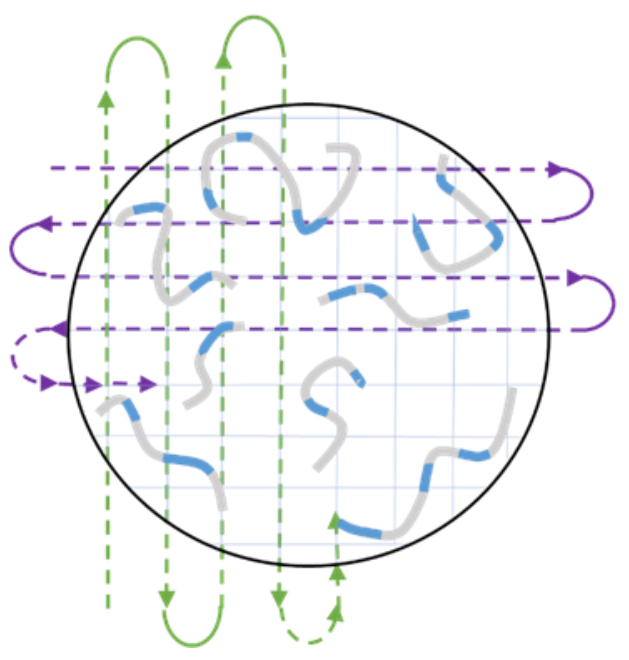

Intersections between

$\%$ Mycorrhizal $=\frac{\text { mycorrhizal roots and gridlines }}{\text { Total }} \times 100$

colonization $=\frac{\text { Total intersections between }}{\text { root and }}$ roots and gridlines 
Figure 2

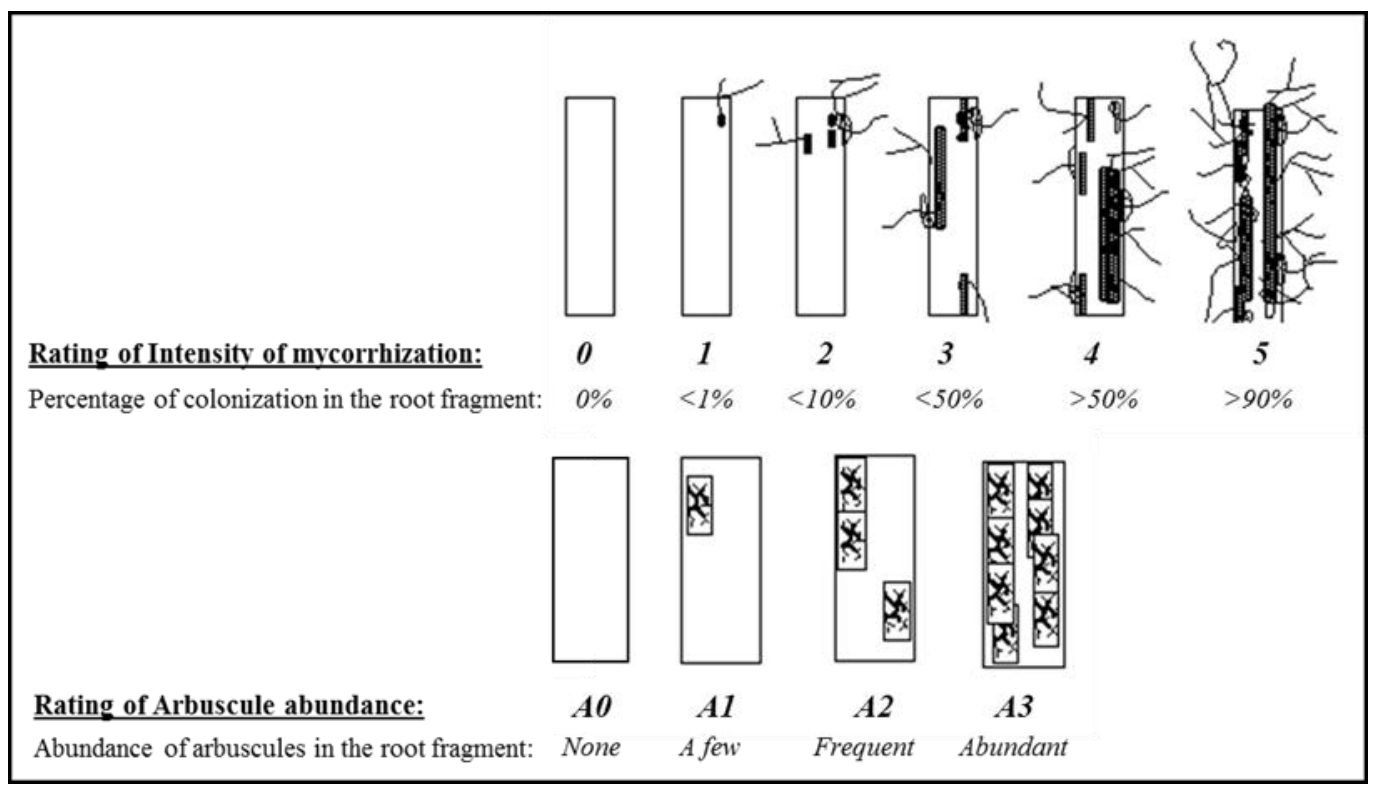


Figure 3

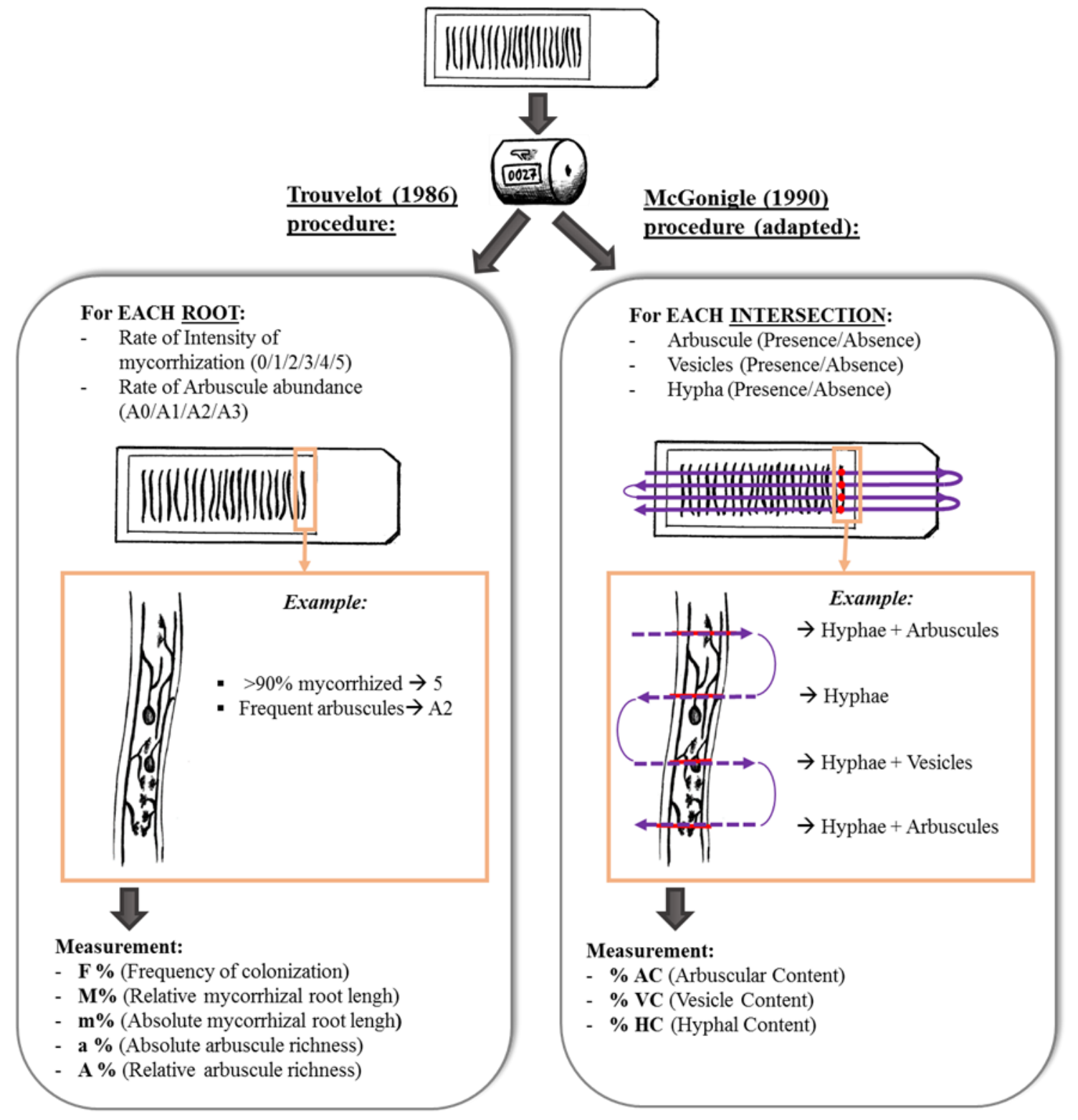

